\title{
La traduction: comparaison entre theories et methodologies
}

Lorella Martinelli*

\section{Les fondements théoriques de la recherche sur la traduction}

À partir des années soixante, l'intérêt pour les phénomènes de traduction occupe une place croissante dans le monde scientifique, surtout dans la culture anglo-saxonne, où commencent à apparaître de vastes recherches succédant aux études dont le caractère intuitif a toujours distingué ce secteur. En particulier, des chercheurs en linguistique manifestent leur forte implication dans ce domaine comme en témoignent les travaux d'Eugène Nida (1964), de John Cunnison Catford (1965), et d'Eugène Nida et Charles Taber (1969).

Les premiers modèles linguistiques de la traduction illustrés par la théorie de Catford et Nida ont souvent été définis comme prescriptifs, quant à leur intention de définir la traduction et le processus de traduction de façon indiscutable et d'établir les règles qui caractérisent la façon correcte de traduire, quels que soient les exemples concrets observés dans la réalité1.

Dans les années soixante-dix, commence à émerger une insatisfaction croissante à l'égard de ces modèles, considérés aujourd'hui comme abstraits et incapables de décrire la réalité traductive. Aux théories prescriptives s'opposent de nouvelles approches descriptives qui se développent au sein d'un mouvement - défini à postériori Manipulation $S c h o o l^{2}$ - se réunissant autour de la proposition de James Holmes (1988) qui entend ouvrir un espace disciplinaire autonome de la recherche sur la traduction, la libérant

\footnotetext{
${ }^{*}$ Università di Chieti-Pescara - Italie

${ }^{1}$ L'approche de Nida fait partie de cette optique reflétant la volonté de contenir les capacités herméneutiques des traducteurs.

${ }^{2}$ Cette dénomination se trouve initialement dans les œuvres de Snell-Hornby (1988) et Gentzler (1993).
} 
de sa dépendance de la linguistique. Le terme Translation Studies attribuable à Holmes reflète un changement d'orientation des études et des recherches dans le domaine de la traduction. L'évolution des Translation Studies consiste initialement en un renforcement du secteur descriptif au détriment des secteurs théorique et appliqué. Le chercheur qui se consacre davantage à la promotion de la section descriptive est sans aucun doute Gideon Toury, représentant de l'école de Tel Aviv. L'intérêt marqué de Toury (1995) pour le secteur descriptif et la dépendance postulée entre les secteurs théorique et descriptif, que l'on peut clairement déduire de sa méthode d'analyse, sont les conditions nécessaires au développement des Translation Studies à partir des années quatre-vingt. Il faut remarquer toutefois que ses réflexions concernent uniquement la traduction littéraire, sans exclure des applications à d'autres réalités traductives. Un élément qui pourrait expliquer la partialité de Toury à l'égard des phénomènes traductifs de nature littéraire/culturelle est la grande influence exercée sur son travail par les théories du sémiologue israélien Itamar Even-Zohar dans les années 1970-1980. Le modèle proposé par ce dernier inscrit au centre de la scène culturelle les phénomènes traductifs, il leur attribue un rôle principal dans l'évolution diachronique de systèmes culturels entiers. Dans cette optique, la traduction n'est plus soumise à une comparaison normative avec l'original, mais elle est considérée comme une catégorie textuelle partiellement autonome, douée de caractéristiques propres que l'on peut relever non seulement au niveau linguistique ou textuel, mais aussi sur le plan de la réception. L'importance de la théorie du polysystème pour la recherche en traduction est indéniable : non seulement elle offre une méthodologie heuristique et un modèle de recherche à la fois systématique et flexible, mais elle met aussi au premier plan la dimension culturelle de la traduction dans le sens le plus large du terme. Toutefois, c'est seulement à la fin des années quatre-vingt que les théoriciens de la traduction ont reconnu la centralité de la nature purement culturelle de la traduction, grâce aux recherches de Susan Bassnett et surtout grâce à la publication du volume de Snell-Hornby Translation Studies : An integrated Approch (1994), dans lequel émerge une nouvelle prise de conscience de la traduction comme processus de transfert interculturel. Au cours des années quatre-vingt-dix, l'attention suscitée par la portée culturelle 
des phénomènes traductifs ne cesse de croître, fruit du travail de quelques spécialistes qui passent peu à peu d'une perspective littéraire traditionnelle à une autre, se rapprochant ainsi davantage des intérêts et des modèles des Cultural Studies. André Lefevere (1992) explore des processus de traduction sur les plans historique et culturel et propose d'élargir la définition du terme «traduction", en la remplaçant par le rewriting. Lefevere est parmi les premiers experts de traduction à manifester un grand intérêt pour les Cultural Studies. Il introduit dans ses réflexions théoriques l'apport humain à travers les concepts de parrainage et d'idéologie, ouvrant la voie à la considération des aspects sociologiques de la traduction, secteur en plein essor de nos jours. L'association des Translation Studies et des Cultural Studies est plus évidente chez l'Américain Lawrence Venuti. Il se montre particulièrement intéressé par les questions de nature éthique, politique, idéologique et économique, et adopte un point de vue historique et culturel qui, à certains égards, se rapproche de Lefevere, mais avec des finalités différentes. Venuti s'oppose à l'opinion traditionnelle qui considère la traduction comme un produit littéraire, ayant un statut inférieur à celui du texte original. Il condamne la pratique de la traduction « naturalisante » et lui oppose la pratique aliénante qui adopte un point de vue éthique à propos $\mathrm{du}$ travail $\mathrm{du}$ traducteur et des rapports entre littératures et cultures différentes. L'originalité de sa pensée consiste dans l'effort de concilier la protection de l'altérité de l'original avec les revendications sur le statut de la traduction et sur celui des traducteurs professionnels. Toutefois, il est très difficile de concilier ces deux positions sans paraître incohérent, comme le sont parfois les conceptions du théoricien américain. Mais si le point de vue historique et culturel de The Translator Invisibility : A History of Translation n'arrive pas à résoudre ces incohérences, les recherches successives (1998) explorent davantage les relations entre la traduction éthique et la traduction idéologique, à travers l'analyse des méthodes et des institutions (droit d'auteur, marché de l'édition, etc...) qui dominent l'activité de la traduction. Le modèle de Venuti peut être considéré comme une ligne de partage permettant d'évaluer l'évolution de la théorie de la traduction au cours d'une quarantaine d'années. Les premières théories des années 60, suivies de l'avènement de la perspective et du déplacement de l'attention sur la 
culture d'arrivée - qui avait caractérisé la naissance des Translation Studies ont été mises en discussion.

L'intérêt pour la traduction, comprise à la fois comme processus et comme produit, a considérablement augmenté au cours de ces dernières années : la tendance est diffuse au niveau international, en témoignent la prolifération des publications dans ce domaine, les nombreux débats et colloques mais aussi l'offre en matière de formation universitaire.

Dans le même temps, le sens de ce terme recouvre une multitude de nouvelles facettes, devenant ainsi une unité de plus en plus complexe. La distinction fondamentale entre la traduction écrite et orale a été suivie par une fragmentation progressive dans des domaines de plus en plus spécialisés: littéraire, technique et / ou scientifique, multimédia, etc. Face aux dimensions de plus en plus macroscopiques engendrées par ce phénomène, on peut se demander si et comment la sectorisation progressive de la traduction représente une évolution positive par rapport à une activité qui joue un rôle clé dans la réalité moderne, toutefois trop rarement valorisée.

Deux réponses possibles peuvent élucider cette problématique : d'une part, les sceptiques qui s'alignent, niant à cette discipline une dimension ontologique. Ils soutiennent qu'il ne s'agit là que d'une pratique auxiliaire, au service d'activités plus importantes dont ils indiquent les modalités et les caractéristiques - par exemple la traduction dite technique ou scientifique propre à certains domaines, comme la médecine, l'économie, le secteur juridique, mais aussi la traduction littéraire au service du monde de la littérature, et ainsi de suite. De l'autre côté des barricades, on trouve ceux qui lisent la fragmentation de la traduction comme un signe positif, indiquant non seulement une augmentation progressive de la visibilité des pratiques de traduction au sens strict dans la société contemporaine, mais aussi et surtout elle révèle le fait que le concept de traduction, en tant que symbole et métaphore de la dimension interculturelle, vient stimuler une grande variété de phénomènes d'aujourd'hui ${ }^{3}$. Ces deux réponses ont un impact significatif auprès de la communauté scientifique internationale, car

\footnotetext{
${ }^{3}$ L'avènement du courant critique déconstructionniste a considérablement contribué à l'utilisation de la traduction dans un sens métaphorique. Pour le philosophe français Jacques Derrida (1985), la traduction est un symbole de l'indétermination essentielle des phénomènes linguistiques.
} 
elles contemplent son statut disciplinaire : est-il légitime de considérer la traduction comme un secteur à part entière, ou la diversification croissante de cette activité doit-elle être comprise comme le signal sans équivoque de sa nature hybride et secondaire par rapport aux disciplines fondamentales, comme la linguistique, la littérature, les études sociologiques, etc ? Cette problématique revient en effet souvent dans la littérature spécialisée et représente la question centrale par excellence dans les discussions concernant l'appareil théorique.

\section{La transversalité disciplinaire}

La traduction a été définie comme une maison avec plusieurs pièces (HATIM, 2001) et l'idée qu'il n'existe qu'une théorie unique et complète a été abandonnée depuis bien longtemps. C'est pourquoi il est de plus en plus courant de parler d'approches à la traduction ou de modèles de recherche. Ces termes soulignent clairement la pluralité des perspectives à partir desquelles il est possible d'expérimenter cette activité. Tout cela va de pair avec les discussions qui illustrent la nature interdisciplinaire ${ }^{4}$ de la traduction, concept qui semblerait résoudre de façon positive toute la problématique de sa nature hybride.

$\mathrm{Si}$, d'une part, il convient de préciser que la transversalité disciplinaire est un thème qui a intéressé, au cours de ces dernières années, un grand nombre de secteurs, d'autre part, on risque de réduire l'appareil théorique à une sorte de spéculation contingente, dont la méthodologie dépend uniquement des conditions matérielles dans lesquelles a eu lieu la pratique de la traduction. Ceux qui la conçoivent tout simplement comme une activité accessoire à d'autres domaines de recherche, objecteront que cette pratique étant très fortement ancrée dans la matérialité de l'expérience qui la renferme, il est impossible d'élaborer une véritable théorie

\footnotetext{
${ }^{4}$ Il est possible d'envisager une multitude d'approches pour la traduction : une approche linguistique, mais aussi une approche sociologique et culturelle. Certains chercheurs étudient les langues et la traduction d'un point de vue politique dans le cadre des politiques linguistiques (CALVET, 1999), d'autres d'un point de vue économique, dans le cadre d'une «économie des langues » (GRIN, 2004). Inutile d'insister sur le fait qu'aucune approche ne saurait épuiser le sujet et que les résultats se complètent davantage puisque ces disciplines contribuent, ensemble, à approfondir la connaissance de la traduction dans toutes ses dimensions.
} 
traductologique, basée sur des hypothèses internes à la discipline. D'où la nécessité de se demander si l'abstraction théorique est totalement impraticable dans une recherche de ce genre et, dans ce cas, nous serions amenés à conclure que traduire est dépourvu de toute valeur scientifique. Peut-être est-ce la raison pour laquelle surgit la nécessité de recourir à des domaines de recherche externes et diversifiés?

Ces interrogations jettent une ombre inquiétante sur la vocation interdisciplinaire de la recherche en traduction qui, tout à coup se révèle comme un parcours obligé pour ceux qui souhaitent travailler dans ce secteur, plutôt que d'être une option, plus ou moins réfléchie. Il n'est donc pas surprenant que le seul élément unissant les chercheurs en traductologie semble être une sorte de stratégie défensive qui les pousse à continuer à célébrer la diversité, surtout au niveau théorique et méthodologique, au détriment de tout concept d'unité de fond $\mathrm{d}^{5}$. Un tel parcours peut s'avérer très risqué pour la crédibilité de la recherche en traductologie.

\section{La traduction comme "pluridiscipline " ou " méta-discipline »}

Une réponse à toutes ces questions est donnée par la manœuvre stratégique de certains spécialistes qui parlent de « pluridiscipline » ou même de « métadiscipline de la traduction ». Selon Jeremy Munday (2001) en effet, les Translation Studies sont le parfait exemple d'un secteur disciplinaire capable de rassembler différentes approches provenant de nombreux domaines linguistiques et culturels, de les modifier et de les réélaborer pour obtenir de nouveaux modèles répondant à la spécificité de leurs propres exigences. La traduction est définie comme une méta-discipline, capable d'accueillir en elle différentes disciplines, avec leurs propres hypothèses théoriques et méthodologiques. Cela serait possible grâce à la capacité « osmotique » de la langue d'absorber la sève (théorique et méthodologique) des autres secteurs et de la transformer en une matière nouvelle, au service de la science de la traduction, prête à répondre à ses besoins spécifiques. Et c'est exactement à travers cette capacité de transformation que réside la caractéristique

\footnotetext{
${ }^{5}$ Henri Meschonnic accorde une grande place à la « poétique de la traduction » qu'il veut libérer du dualisme opposant la théorie à la pratique. Il n’y a plus d'antagonismes entre la théorie et la pratique mais une homogénéité dialectique (1973).
} 
principale autour de laquelle ce secteur disciplinaire pourrait se recentrer : que la traduction ait la capacité de transformer le texte source en une autre matière, non seulement du point de vue linguistique mais aussi idéologique, politique, économique, etc... semble une considération difficile à nier. La traduction est donc le résultat d'un mouvement configurant également une polarité. D'une part, le texte à traduire, de l'autre, le texte traduit. L'un et le multiple. L'œuvre en soi vit une unicité qui est aussi la garantie de son inviolabilité et de son autonomie. Imaginer Le Rouge et le Noir différent serait impossible, comme il serait impossible de croire que quelqu'un puisse réécrire un autre Le Rouge et le Noir, capable d'évincer l'original. Le texte de Stendhal pourra être copié, réécrit et adapté, mais rien ne pourra venir égratigner son unicité. Cela ne signifie pas qu'il est illégitime de copier, plagier ou réécrire un chef-d'œuvre. En effet, à bien des égards, l'essence de la culture consiste précisément en cela - en un dialogisme permanent qui, en se déployant de façon diachronique et synchronique, met en œuvre toutes les potentialités inhérentes à l'échange entre le texte qui donne et le texte qui reçoit. Ce processus dynamique implique l'opposition un / multiple dans le sens où une œuvre réussit à vivre dans le temps, grâce à son unicité qui se transforme en multiplicité. Et c'est cette transformation qui met en cause l'appropriation du texte par le lecteur. Interpréter équivaut donc à traduire, justement parce que chaque événement herméneutique est toujours un mouvement vers, un voyage au-delà de $1^{\prime}$ objet et de son essence ${ }^{6}$. D'où l'intégration, l'assimilation et l'inscription du nouveau texte dans le système de la culture qui, en acceptant sa valeur sémantique expressive, l'insère dans la grande dynamique du faire et du défaire de ses constructions sémiotiques.

\section{Les itinéraires herméneutiques de la traduction}

Le postulat est que l'œuvre d'art peut engendrer une succession d'interprétations qui, en assurant sa vie dans le futur, va témoigner, dans leur multiplicité, de l'unicité sur laquelle se fondent les raisons de sa

\footnotetext{
${ }^{6}$ Marianne Lederer résume en ces termes les principaux acquis de la théorie interprétative de la traduction : «La théorie interprétative a établi que le processus [de traduction] consistait à comprendre le texte original, à déverbaliser sa forme linguistique et à exprimer, dans une autre langue, les idées comprises et les sentiments ressentis » (1994, p. 11).
} 
persistance au cours du temps. Comment ne pas lire la vérité inhérente dans cette totalité esthétique dont l'œuvre est l'expression ? Sans cette vérité aucune œuvre n'est en mesure de générer ce que Gadamer appelle la fusion des horizons en tant que lieu de rencontre entre sujet et objet. Mais il est clair que toute interprétation étant temporaire et éphémère, elle suppose une lecture anti-dogmatique par rapport à la Wahreit dont tout chef-d'œuvre se nourrit. Sur la base de la fermeture de la grande œuvre se construit l'ouverture appartenant aux mille réponses critiques que suscite un chefd'œuvre. Paradoxalement, les itinéraires herméneutiques qui se détachent d'elle - et qui, dans son dessin textuel, sont compris de façon pragmatique révèlent cette vocation à se tendre vers une autre vie, vers un mot qui va audelà du mot original. C'est l'histoire de la culture qui apparaît toujours, en tant que rencontre de systèmes, comme échange incessant d'informations qui vont tracer un vaste réseau articulé, sur lequel d'autres œuvres - uniques et inviolables elles aussi - seront construites pour assurer d'autres échanges sémiotiques. D'où le déploiement de nouveaux parcours qui, sans jamais interrompre le voyage infini de la culture, continueront à vivre dans de nouvelles interprétations, sans relâche, sans jamais fermer la porte à d'autres voix et à d'autres dialogues possibles. C'est ainsi que la culture devient l'espace où s'exprime, dans un travail intense et en partie imprévisible, un processus de traduction qui vacille entre l'unicité textuelle et la multiplicité des réponses possibles, entre la voix individuelle et la voix collective, entre le dialogue silencieux du sujet avec lui-même et le dialogue perçu comme un crépitant chaos informe de voix. Culture donc, comme grande machine de la traduction. L'un et le multiple, l'original et ses traductions, le texte source et le texte cible. Si le discours sur l'opposition entre l'unité textuelle et ses interprétations a un sens, nous pouvons alors supposer qu'il existe une opposition entre l'unicité du texte et la virtualité des traductions possibles. Née dans un contexte spécifique, l'œuvre d'art se place toujours au-delà de son moment historique qui, jamais vraiment oublié, a une fonction secondaire par rapport à la signification et à la valeur métahistorique qu'elle possède. La traduction vit donc grâce à son historicité : elle naît avec l'époque de son écriture et sera inévitablement évincée - par pur effet de la dynamique de la culture et non pas par une hiérarchie des valeurs - par la 
traduction plus récente, ou plus proche de la culture des lecteurs pour qui ce travail a été adapté et fait. Comme l'a souligné Walter Benjamin (1923), tandis que la parole du poète survit dans sa propre langue, même la plus importante des traductions est destinée à entrer (et à être absorbée) dans le développement de la langue, et à périr dans son renouvellement.

Ce fait irréfutable rend encore plus problématique et « ouverte » la tâche du traducteur qui, au moment où il conclut le processus de recodification de la langue source à la langue cible, sait parfaitement qu'un autre traducteur est probablement en train de répéter son travail sur le même texte, mais avec l'avantage de lui succéder. Dans le processus de traduction, il est clair qu'il s'agit là, sur le plan linguistique, d'un privilège qui se fonde sur la proximité entre le traducteur et l'éventuel lecteur. Cet atout demeure malgré une augmentation croissante de la distance entre le texte traduit et le texte original.

Or, il va de soi que la traduction est dépositaire d'une historicité en tant qu'interprétation liée à un moment précis, tandis que le texte a une collocation qui, pour être métahistorique, est une non collocation. C'est une traversée temporelle qui toutefois ne peut exclure une historicité ontologiquement différente de celle de la traduction. En d'autres termes, une œuvre d'art naît dans un moment de l'Histoire pour s'abandonner cependant seulement à son flux et à son devenir. Cela est différent pour l'interprétation / traduction qui est toujours historiquement déterminée, et en tant qu'enregistrement d'un moment, elle est donc provisoire (PRETE, 2011).

Traduire est interpréter ${ }^{7}$ : chaque traduction enregistre, en définitive, une vérité qui ne peut être semblable à celle de l'objet interprété, étant donné que l'historicité du texte traduit localise et délimite le moment spécifique d'une vérité conquise. Elle apparaît alors comme une tentative de s'approcher de l'horizon de l'auteur et du lecteur dans un projet qui, en se servant des attentes positives de l'expérience herméneutique, met au premier plan le langage, défini par Heidegger comme ce que l'homme trouve

\footnotetext{
${ }^{7}$ Voir à ce propos George Steiner quand il affirme que tout acte traductif a la valeur d'une interprétation (1975).
} 
dans son voisinage immédiat. D'une certaine manière le traducteur sait qu'il se déplace dans la multitude mais il considère son propre travail comme une expérience placée sous le seuil de l'unicité. Nos traductions sont des expériences uniques sur un territoire historiquement marqué par les victimes de tant de traductions du même hypotexte. En effet, chaque expérience de l'acte de traduire est une expérience différente et unique, dans laquelle le traducteur transpose son individualité et sa sensibilité plus ou moins inavouée. Ainsi, historicité de l'interprétation / traduction mais aussi prise de conscience de la possibilité de traduire ce qui, dans une vision statique des systèmes culturels, pourrait être défini comme étant intraduisible. Dans une perspective sémiotique-culturelle, on ne parle jamais de l'intraduisible, mais au contraire, d'une traduction incessante qui implique la dynamique de la rencontre et du conflit des cultures et de la négociation (ECO, 2003; FORTINI, 2011) ou la dynamique des influences et de la résistance aux influences. L'histoire de la culture de chaque nation peut être analysée sous deux points de vue : d'une part comme développement immanent, d'autre part comme le résultat d'influences multiformes externes. (LOTMAN, 1993). Ces deux processus sont étroitement liés et leur séparation n'est possible que dans les modalités de l'abstraction scientifique. L'activité de traduction, où qu'elle soit, s'inscrit dans cet univers qui, en constante métamorphose, exprime à chaque instant la double pratique de l'interprétation / traductive. Des mots qui se rencontrent et qui veulent être lus et interprétés, des mots qui interprètent et qui, à leur tour, au moment même où ils traduisent, supposent une interprétation ultérieure. Et tout cela a lieu sur une plate-forme sémantique où, dans le rapport entre la partie et le tout, entre l'un et le multiple, vit l'échange fait de textes qui influent sur le temps et en sont influencés (STEINER, 1975).

Peut-être serait-il possible d'étendre cette capacité inhérente à l'acte de traduction de transformer le texte source également théoriquement et de soutenir donc que la recherche, dans cette discipline, utilise des outils empruntés à d'autres domaines disciplinaires, mais qu'elle les transforme en les faisant siens, afin de satisfaire les exigences des spécialistes des phénomènes de traduction? 
Sa capacité à modifier, à modeler le matériel de départ (le texte original auquel nous pourrions ajouter les théories et les méthodes empruntées aux disciplines analogues) dans de nouvelles entités, a été assimilée à la fonction performative du langage où la langue devient ellemême une action. Les pratiques de la traduction, en effet, mettent en circulation non seulement une série de signifiés qui gisaient inertes dans le matériel de départ, atteignant des puissances inexprimées, mais elles sont elles-mêmes des déclarations d'intention, par le simple fait de se présenter comme des traductions.

Les textes se définissant comme tels ont clairement une réception différente par rapport au soi-disant texte d'origine, comme on peut aisément le déduire d'après certaines données du secteur de l'édition d'aujourd'hui, même de l'édition dite artistique ${ }^{8}$. La traduction comme processus et comme produit se caractérise donc comme une pratique sociale et textuelle reconnue, unique, dotée d'une propre autonomie. Selon Antony Pym (2000), c'est l'une des nombreuses modalités de communication interculturelle qui se distingue des autres parce qu'elle implique des investissements précis en termes de formation, de temps et de coûts. Étendre sans distinction la définition de traduction à toutes les pratiques textuelles existantes (en respectant le principe déconstructionniste de l'inexistence de l'originalité absolue) est très nuisible pour la traduction elle-même, car cela annule sa singularité. La spécificité indéniable du domaine traductologique (même dans sa diversification actuelle) prévoit et nécessite une réélaboration massive des théories ou des modèles de recherche qui peuvent être empruntés par des disciplines analogues. Cette notion est évidente en matière de recherche en traduction à travers l'utilisation des corpus, mais aussi l'adaptation des théories systémiques, de dérivation sémiotique et littéraire, ou le secteur de la qualité de la traduction utilisant les présupposés linguistiques de Michael Halliday, qui constituent de bons exemples.

\footnotetext{
${ }^{8}$ Les études sur le concept et la fonction des pseudo-traductions ou des traductions fictives (textes qui se présentent comme des traductions même si ce sont des originaux) représentent un exemple très intéressant de l'efficacité du concept de traduction et de la manière dont elle pourrait servir plusieurs intérêts. Cf. Bassnett, 1980; Toury, 1995.
} 


\section{Conclusion}

La traduction, comme toute autre discipline récemment créée, a élaboré, dans un premier temps, ses modèles de recherche dans un système «écologique» essayant d'utiliser au mieux et d'adapter à ses propres finalités des théories et des méthodologies préexistantes de provenance externe. C'est ainsi qu'elle a réussi à les perfectionner, en élaborant des modèles de plus en plus spécifiques qui peuvent être diffusés aujourd'hui vers d'autres disciplines ${ }^{9}$; par conséquent, si la reconnaissance de la dette des études en traduction envers les disciplines analogues est un acte dû, il est maintenant temps de faire le point et d'attribuer plus de visibilité au parcours effectué par ce secteur, en consolidant ses progrès, en particulier dans les domaines théorique et méthodologique. Aujourd'hui une nouvelle époque pour la recherche sur la traduction commence: époque de l'abolissement des frontières disciplinaires et de la diversification théorique et méthodologique qui répond aux besoins toujours plus complexes de notre société, mais aussi à l'importance qu'assument les phénomènes de la communication interculturelle. À travers une analyse diachronique minutieuse, il en découle une vue d'ensemble dynamique de réciprocité intersectorielle, qui attribue aux théories de la traduction une position de grande envergure dans le contexte de l'évolution des disciplines socio-humanistes.

\section{Bibliographie}

BALLARD, Michel. Histoire de la traduction. Repères historiques et culturels. Bruxelles: De Boeck, 2013.

BASSNETT, Susan. Translation studies. London \& New York: Routledge, 1980.

\footnotetext{
${ }^{9}$ Susan Bassnett est la première spécialiste à considérer la capacité des Translation studies à exporter des modèles de recherche. Elle soutenait, dans Comparative Literature: A critical Introduction (1993), que cette nouvelle discipline influerait fortement sur l'évolution de la littérature comparée. Par la suite (1998), la chercheuse a relevé des affinités et des contacts essentiellement au niveau méthodologique aussi avec les Cultural Studies. Kristen Malmkjær (1999), quant à elle, a plutôt insisté sur la réciprocité entre la linguistique et les Translation Studies.
} 
Comparative literature: a critical introduction. Oxford: Black-well, 1993.

BASSNETT, Susan; LEFEVERE, André. Constructing cultures: essays in literary translation. Clevedon: Multilingual Matters, 1998.

BENJAMIN, Walter. La tâche du traducteur. In: Cuvres I, traduit de l'allemand par Maurice de Gandillac. Paris: Gallimard, [édition originale en allemand 1923] 2000.

CALVET, Louis-Jean. La guerre des langues et les politiques linguistiques. Paris: Hachette, 1999.

CATFORD, John Cunnison. A linguistic theory of translation. London: O.U.P., 1965.

DERRIDA, Jacques. Des Tours de Babel. Psyché, Paris: Galilée, 1987.

ECO, Umberto. Dire presque la même chose, traduit de l'italien par Bouzaher, Myriem. Paris: Grasset, [édition originale en italien 2003] 2006.

EVEN-ZOHAR, Itamar. Papers in historical poetics. Tel Aviv: The Porte Institute, 1978.

FORTINI, Franco. Lezioni sulla traduzione. Macerata: Quodlibet, 2011.

GENTZLER, Edwin. Contemporary translation theories. London \& New York: Routledge, 1993.

GRIN, François. On the costs of linguistic diversity. In: VAN PARIJS, Philippe. Linguistic diversity and economic solidarity. Bruxelles: De Boeck, 2004, p. 193-206.

HATIM, Basil. Teaching and researching translation. London: Pearson Education, 2001

HEIDEGGER, Martin. Acheminement vers la parole, traduit de l'allemand par Beaufret, Jean Brokmeier, Wolfgang et Fédier, François. Paris: Gallimard, [édition originale en allemand 1959] 1976.

HOLMES, James S. Translated! Papers on literary translation and translation studies. Amsterdam: Rodopi, 1988.

LADMIRAL, Jean-René, Traduire: théorèmes pour la traduction. Paris : Gallimard, 1994.

LEDERER, Marianne. La traduction aujourd'hui. Paris: Hachette, 1994.

LEFEVERE, André. Translation, rewriting and the manipulation of literary fame. London \& New-York: Routledge, 1992. 
LOTMAN, Jury. L'explosion et la culture, traduit du russe par Merkoulova, Inna. Limoges : Presses Universitaires de Limoges, [édition originale en russe 1992] 2005.

MALMKJAER, Kirsten. Translation and linguistic. Textus, 12, p. 263-273.

MESCHONNIC, Henri. Pour la poétique II. Épistémologie de l'écriture poétique de la traduction. Paris: Gallimard, 1973.

NIDA, Eugène. Toward a science of translation. Leiden: Brill, 1964.

NIDA, Eugène. TABER, Charles. The theory and practice of translation. Leiden: Brill, 1969.

MUNDAY, Jeremy. Introducing translation studies: theories and applications. London \& New-York: Routledge, 2001.

PERGNIER, Maurice, Les fondements socio-linguistiques de la traduction. Paris: Les Belles Lettres, 2017.

PRETE, Antonio. All'ombra dell'altra lingua. Per una poetica della traduzione. Torino: Bollati Boringhieri, 2011.

PYM, Antony. On Cooperation. In: OLOHAN, Maeve. Intercultural faultlines. Manchester: St. Jerome, 2000, p. 181-192.

SNELL-HORNBY, Mary; POCHHACKER, Franz \& KAINDL, Klaus. Translation Studies: an interdiscipline. Amsterdam \& Philadelphia: John Benjamins, 1994.

STEINER, George. Après Babel. Une poétique du dire et de la traduction, traduit de l'anglais par Lotringer Lucienne. Paris: Albin Michel, [édition originale en anglais 1975] 1978.

TOURY, Gideon. Descriptive Translation Studies and beyond. Amsterdam \& Philadelphia: John Benjamins, 1995.

VENUTI, Lawrence. The translator's invisibility: A history of translation. London \& New York: Routledge, 1995.

The scandals of translation. Toward an ethics of difference. London \& New-York: Routledge, 1998.

\section{Résumé}

La traduction a toujours été caractérisée par une multiplicité et une variété de méthodologies pour atteindre des objectifs diversifiés. Il y a quelques années, on discutait essentiellement du caractère interdisciplinaire de la 
traduction, or on préfère aujourd'hui la définir comme une «multidiscipline», terme qui entend souligner non seulement l'autonomie disciplinaire de ce secteur, récemment consolidée, mais aussi la pluralité des approches et des perspectives qui la caractérisent. À partir d'une minutieuse analyse diachronique, se profile une vue d'ensemble dynamique de réciprocité intersectorielle attribuant aux théories de la traduction une position de grande envergure qui concerne l'évolution des sciences humaines et sociales.

Mots clés: Traduction; Création; Confluences; Pluralité; Négociation.

\section{Resumo}

A tradução sempre se caracterizou pela sua variedade e multiplicidade de metodologias utilizadas para alcançar diferentes objetivos. Se até há pouco tempo discutia-se sobre a postura essencialmente interdisciplinar da tradução, hoje é preferível defini-la como uma "multidisciplina", termo que pretende sublinhar quer a autonomia disciplinar do setor, recentemente consolidada, quer a pluralidade de abordagens e perspectivas que a distinguem. O que se revela, a partir de uma análise diacrônica, é um animado panorama de trocas intersetoriais que atribui à(s) teoria(s) da tradução uma posição de grande relevância dentro do contexto da evolução das disciplinas humanísticas e das ciências sociais.

Palavras-chave: Tradução; Criação; Confluências; Pluralidade; Negociação. 